ÍNDICE DE ESPECIALIZACIÓN PUNTO BASE Y COEFICIENTE DE ESPECIALIZACIÓN: ANÁLISIS COMPARATIVO Y ESTADÍSTICO

\title{
ÍNDICE DE ESPECIALIZACIÓN PUNTO BASE Y COEFICIENTE DE ESPECIALIZACIÓN: ANÁLISIS COMPARATIVO Y ESTADÍSTICO
}

\section{THE BASE POINT SPECIALIZATION INDEX AND THE COEFFICIENT OF SPECIALIZATION: COMPARATIVE AND STATISTICAL ANALYSIS}

\begin{abstract}
Mario Gutiérrez Lagunes*, José Manuel Romo Orozco**, Aderak Quintana Estrada***
\end{abstract}

*Profesor Investigador de la Universidad Autónoma de San Luis Potosí. Unidad Académica Multidisciplinaria de la Zona Media.

**Profesor Investigador de la Universidad Autónoma de San Luis Potosí. Unidad Académica Multidisciplinaria de la Zona Media.

***Profesor Investigador de la Universidad Autónoma de San Luis Potosí. Unidad Académica Multidisciplinaria de la Zona Media.

Dirección para recibir correspondencia: aderak.quintana@uaslp.mx 
ÍNDICE DE ESPECIALIZACIÓN PUNTO BASE Y COEFICIENTE DE ESPECIALIZACIÓN: ANÁLISIS COMPARATIVO Y ESTADÍSTICO

\section{RESUMEN}

OBJETIVO: Presentar la metodología del índice de especialización punto base (IEPB), que puede ser utilizada para establecer tendencias de actividades productivas con suficiente precisión, para ser comparado con el coeficiente de especialización (CE), reportado por la literatura especializada.

MATERIAL Y MÉTODO: La presente investigación es de tipo descriptivo, explicativo y estadístico; descriptivo debido a que se identifican las principales características de ambos índices al ser usados con datos del sector manufacturero mexicano, comparativo ya que se encuentran características y diferencias que presentan cada uno, y estadístico porque al realizarse una prueba de hipótesis de muestras apareadas se identifica similitud entre ambos índices.

RESULTADOS: Al aplicarse el IEPB y CE a los diferentes subsectores del sector manufacturero mexicano para establecer tendencias se pudieron observar comportamientos similares; a partir de la prueba estadística realizada, ambas metodologías arrojan resultados similares.

CONCLUSIÓN: El análisis demuestra que ambos índices, el IEPB y CE, son estadísticamente indistintos. Además existe una relación de complementariedad, sobre todo cuando son usados en análisis sectoriales, ya que el primero se ve reforzado y ampliado cuando se recurre al segundo índice.

PALABRAS CLAVE: Especialización. Análisis sectorial. Desempeño económico.

\section{ABSTRACT}

OBJECTIVE: To present the methodology of the Base Point Specialization Index (IEPB), which can be used to establish trends in productive activities with enough precision, to be compared with the Coefficient of Specialization (CE), reported by the specialized literature.

MATERIAL Y METHOD: This investigation is descriptive, explanatory and statistical; descriptive because the main characteristics of both indexes are identified when they are used with data 
ÍNDICE DE ESPECIALIZACIÓN PUNTO BASE Y COEFICIENTE DE ESPECIALIZACIÓN: ANÁLISIS COMPARATIVO Y ESTADÍSTICO

from the Mexican manufacturing sector, comparative since there are characteristics and differences that each one presents and statistical because when a hypothesis test of paired samples is made, the similarity between both indexes can be identified.

RESULTS: When applying the IEPB and CE to the different subsectors of the Mexican manufacturing sector to establish trends, similar behaviors could be observed and based on the statistical test performed, both methodologies show similar results.

CONCLUSION: The analysis shows that both indexes, the IEPB and CE, are statistically indistinct. There is also a complementary relationship, especially when they are used in sectoral analyzes, since the IEPB is reinforced and expanded when the CE is used.

KEYWORDS: Specialization. Sectoral analysis. Economic performance.

\section{INTRODUCCIÓN}

El objeto de este trabajo, es presentar la metodología del IEPB, propuesta por GutiérrezLagunes, (2010, 2012, 2014a y 2014b), que puede ser utilizada para establecer tendencias de actividades productivas con suficiente precisión. Mediante procedimientos descriptivos y estadísticos, se le compara con el CE, reportado por la literatura especializada. Más allá de señalar ventajas y desventajas de los mismos, el propósito es destacar las principales características y diferencias que tiene cada uno, resaltando su complementariedad.

Estas metodologías pueden ser aplicadas a variables de distinta índole. Para realizar el análisis comparativo, en este caso se consideraron datos del sector manufacturero mexicano, empleando información trimestral de los 21 subsectores que componen dicha actividad productiva, durante el periodo comprendido del 2000-2015; ambas dan cuenta de manera general del comportamiento que cada subsector tuvo durante este periodo, el IEPB registra el mejor o peor desempeño de cada uno, y el CE da muestra de la especialización o subespecialización manufacturera. Al considerarlas como complementarias, la información obtenida proporciona un mejor panorama del comportamiento general de las actividades. 
ÍNDICE DE ESPECIALIZACIÓN PUNTO BASE Y COEFICIENTE DE ESPECIALIZACIÓN: ANÁLISIS COMPARATIVO Y ESTADÍSTICO

El trabajo se divide en cinco partes, la primera plantea de manera general la importancia que tienen los coeficientes absolutos en los estudios sectoriales y regionales, posteriormente, la segunda presenta la metodología del IEPB, que se acompaña del análisis de los resultados aplicados a los subsectores de la manufactura en México, asimismo, la tercera aborda la metodología del CE, en la cuarta se presenta la discusión de los resultados; y, por último, se encuentran las reflexiones finales.

\section{Medidas de desempeño}

El análisis de la situación actual requiere la identificación de problemas y potencialidades, dimensionando los obstáculos y oportunidades que existen en una realidad compleja. Ante las asimetrías, es indispensable adoptar medidas que las mitiguen, y es aquí donde los estudios sectoriales y regionales juegan un papel esencial.

En el diagnóstico global de una economía, se tiene como propósito medir el desempeño alcanzado por sus localidades (o actividades), pero en menor escala pueden existir análisis parciales que caractericen a determinada actividad. El escenario deseable en estos análisis es que cada sector productivo muestre un desempeño acorde con las potencialidades territoriales, así como a la política económica inscrita en el contexto nacional e internacional.

La gestión de la información permite establecer, evaluar y corregir las estrategias seguidas, de manera que teniendo en cuenta su disponibilidad, se recomienda utilizar indicadores que permitan caracterizar las áreas de oportunidad. En este ejercicio, se reconoce que el uso de herramientas y de un marco conceptual común facilitan no sólo la transformación de datos en información útil, sino también la elaboración de estrategias para la elaboración de políticas y su planificación (Sepúlveda, 2008).

Quiroga (2001) define a los indicadores como variables que, en función del valor que asumen temporalmente, despliegan significados que los usuarios decodifican más allá de lo que muestran directamente y que permiten hacer juicios sobre las condiciones del sistema. Sin embargo, el uso de indicadores puede ser de difícil interpretación, por lo cual es frecuente su integración en índices o coeficientes. 
ÍNDICE DE ESPECIALIZACIÓN PUNTO BASE Y COEFICIENTE DE ESPECIALIZACIÓN: ANÁLISIS COMPARATIVO Y ESTADÍSTICO

Presentados por diversos investigadores, los coeficientes exhiben de manera general la función que desempeña cada sector productivo, o unidad espacial, en un contexto macro, temporal o espacial, que se toma como referencia. Con su aplicación se determina el papel de cada unidad de análisis, lo que permite identificar su influencia en la división del trabajo y en su especialización. Para el cálculo de estos coeficientes se suelen emplear indicadores, tales como: el producto interno bruto y la población económicamente activa, entre otros (Méndez y Yizhou, 2007).

La especialización puede ser vista como un concepto que describe la manera en que se distribuye una variable, como la producción o el comercio exterior, en un conjunto. Su estimación muestra la similitud de la estructura económica inscrita en un patrón de comparación y se utiliza como medida de la especialización bajo el supuesto de que la distribución de referencia se diversifica en términos relativos. Según Cuadrado-Roura y Maroto-Sánchez (2012), "la especialización regional puede constituir un factor explicativo del crecimiento económico regional (aunque considerando siempre el 'efecto nacional') y, por supuesto, de la posible convergencia de las regiones de un país en términos de productividad y de ingresos por habitante".

En esta línea, la literatura especializada distingue dos grupos de indicadores: los absolutos, que reflejan si la especialización esta sesgada hacia una industria o rama en particular sin tomar en cuenta la distribución espacial, y los relativos, que muestran comparativos de la estructura productiva o comercial de distintas unidades territoriales, tratando de cuantificar el grado de similitud o divergencia de regional. En este segundo caso, un incremento en el índice indica un incremento de las disparidades en la estructura productiva del territorio analizado (Gordo y Pérez, 2003).

En esta investigación el IEPB, inscrito en la línea de coeficientes absolutos, y el CE son aplicados a las distintas actividades que componen al sector manufacturero nacional para estimar que tan homogéneo ha sido su comportamiento en años recientes, para así poder determinar sus diferencias y resaltar su complementariedad. 
ÍNDICE DE ESPECIALIZACIÓN PUNTO BASE Y COEFICIENTE DE ESPECIALIZACIÓN: ANÁLISIS COMPARATIVO Y ESTADÍSTICO

\section{MATERIAL Y MÉTODO}

\section{Índice de Especialización Punto Base (IEPB)}

El índice de especialización punto base (IEPB) forma parte del Factor de Crecimiento Económico (Gutiérrez-Lagunes, 2010, 2012, 2014a y 2014b), el cual fue conceptualizado desde sus inicios para analizar la industria exportadora y su contribución a cada una de las ramas económicas. Este índice arroja información sobre la participación que tiene cada unidad estudiada en la totalidad durante un periodo específico; es decir, es el comportamiento de la actividad $\mathrm{j}$ en el conjunto de $\mathrm{n}$ actividades en el periodo $\mathrm{i}$.

La expresión matemática que define al IEPB, aplicado a los subsectores manufactureros como parte de la dinámica económica nacional, es:

$$
I_{E P} B_{i j}=\frac{\frac{V_{i j}}{\sum_{i=1}^{n} V_{i j}}}{\sum_{j=1}^{m} V_{i j}}
$$

Donde:

$I E P B_{i j}=$ Índice de especialización de la actividad j en el periodo i.

$V_{i j}=$ totalidad en el periodo i por actividad $\mathrm{j}$.

$\sum_{i=1}^{n} V_{i j}=$ totalidad de la actividad $\mathrm{j}$.

$\sum_{j=1}^{m} V_{i j}=$ totalidad en el periodo i.

$\sum_{j=1}^{m} V_{1 j}=$ totalidad del Año 1.

El IEPB se aplicó al sector manufacturero mexicano, para ello se utilizó información trimestral de los 21 subsectores que componen dicha actividad productiva, se consideró como base el periodo del 2000 al 2015 (INEGI, 2017).

La metodología de este índice compara cada subsector manufacturero (actividad j) del PIB con toda la economía del sector manufacturero (totalidad) desde un año base; así, un subsector GUTIERRÉZ-LAGUNES M., ROMO-OROZCO J. M., QUINTANA-ESTRADA A. 
ÍNDICE DE ESPECIALIZACIÓN PUNTO BASE Y COEFICIENTE DE ESPECIALIZACIÓN: ANÁLISIS COMPARATIVO Y ESTADÍSTICO

económico es considerado de menor desempeño en la medida en que la evolución de su indicador resultase inferior al de la economía en su conjunto, y es de mayor desempeño, si el subsector económico tiene un indicador superior al promedio.

\section{Análisis de los resultados - IEPB}

Los principales resultados del IEPB para el periodo i, cuando se aplica al PIB del sector manufacturero, fueron:

- De la comparación de los 21 subsectores, ocho de ellos tuvieron mejor índice promedio que la media general de 0.958 (ver figura 1).

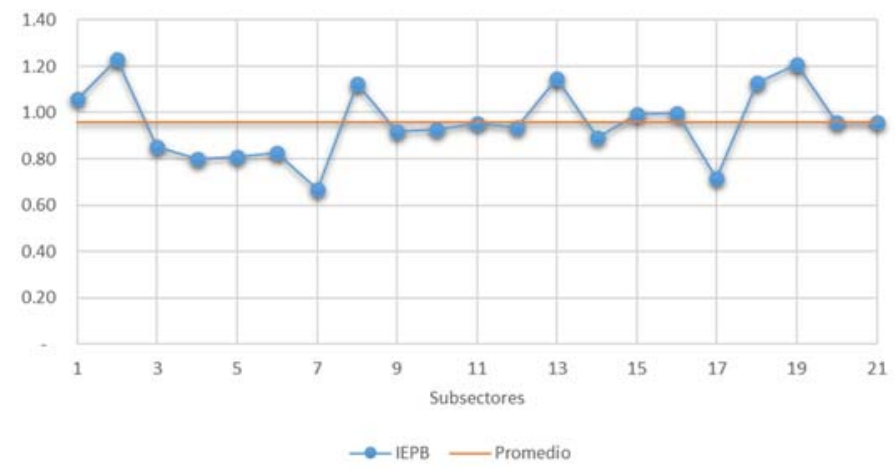

Figura 1. Desempeño de subsectores según IEPB.

Fuente: Elaborado con cálculos de información obtenida del INEGI, 2017.

- En función del promedio trimestral, el mejor desempeño se logró en el tercer trimestre del 2000 (1.021) y el peor desempeño fue en el primer trimestre del 2014 (0.904).

- El mejor subsector fue el 336: Fabricación de equipo de transporte (1.752), alcanzado en el 1 trimestre del 2015. El peor subsector fue el 321: Industria de la madera (0.568) en el cuarto trimestre del 2008.

- En la figura 2, se observa la participación que tiene cada subsector en el conjunto del crecimiento económico nacional. De acuerdo al promedio de estos coeficientes, el mejor desempeño se da en el subsector 312: Industria de las bebidas y del tabaco (1.231), y el peor promedio ocurre en el subsector 321: Industria de la madera (0.669). Adicionalmente, este último subsector fue el que obtuvo el mínimo histórico (cuarto trimestre del 2008), y el 
peor desempeño promedio de los periodos, aconteció en el primer trimestre del 2014. Se hace notar que el subsector 336: Equipo de transporte, obtuvo el mejor coeficiente de especialización del periodo en estudio (1.752); a partir de su caída en el segundo trimestre de 2009 , ha tenido una tendencia positiva sostenida.

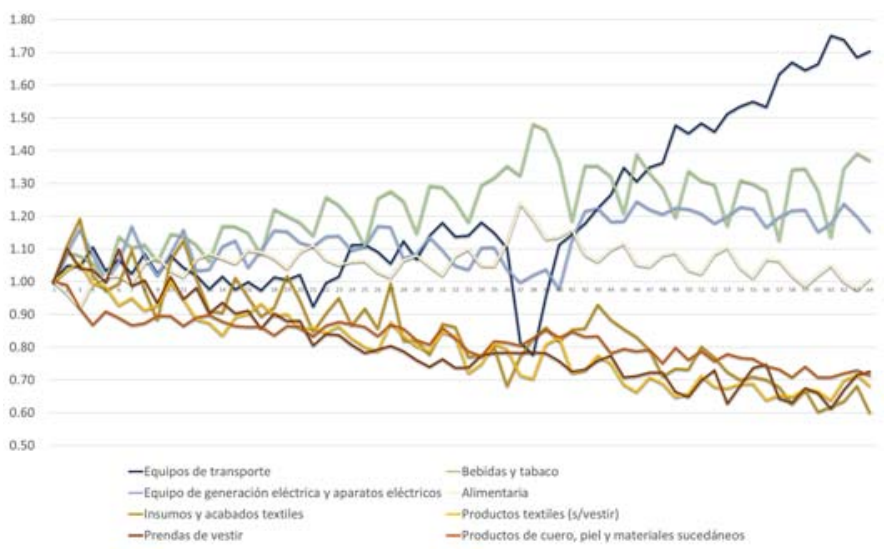

Figura 2. IEPB México, selección de subsectores de la actividad manufacturera. Información trimestral, 2000-2015.

Fuente: Elaborado con información obtenida del anexo, cuadro 1.

En general, el IEPB aplicado al sector manufacturero registra un comportamiento acorde con el papel que ha jugado dicho sector en la vida económica nacional, ya que en las últimas cuatro décadas esta actividad ha sufrido importantes transformaciones en su estructura económica. Los principales cambios en este sector se aceleran con la apertura de la economía nacional iniciada a mediados de los ochenta, se agilizan con la entrada en vigor del Tratado de Libre Comercio de América del Norte (TLCAN), bajo este contexto se ve modificada su estructura comercial, productiva, de inversión y empleo, y algunas ramas consideradas como tradicionales son desplazadas por otras más dinámicas (CEFP, 2005, pp. 6-7).

De acuerdo a los resultados que se presentan en el cuadro 1 del anexo, es posible observar como los subsectores que registran una mejor dinámica en el crecimiento económico son las actividades que están orientados al mercado externo y a la producción de bienes de consumo duradero, principalmente el subsector 335 (fabricación de equipo de generación eléctrica y aparatos eléctricos) y 336 (fabricación de equipo de transporte), fuertemente vinculados a la dinámica del comercio exterior del país, como es de esperar, dada la dependencia económica a 
ÍNDICE DE ESPECIALIZACIÓN PUNTO BASE Y COEFICIENTE DE ESPECIALIZACIÓN: ANÁLISIS COMPARATIVO Y ESTADÍSTICO

los mercados externos, los vaivenes económicos arrastran a este tipo de actividades, como fue durante el periodo más difícil de la crisis de 2008.

Otros subsectores que destacan en su desempeño son los de consumo final no duradero, como el subsector 311 (industria alimentaria) y 312 (industria de las bebidas y del tabaco). El comportamiento observado en estos dos subsectores es reflejo de la dinámica de la consolidación que se ha dado en los últimos años entre los grandes corporativos de propiedad nacional y extranjera, configurando mercados que tienden a la fuerte concentración (Vázquez, 2014, pp. 55-61).

Es de llamar la atención las actividades vinculadas a la industria textil y del vestido como los subsectores 313 (fabricación de insumos y acabados textiles), 314 (fabricación de productos textiles), 315 (fabricación de prendas de vestir) y 316 (curtido, acabado y fabricación de productos de cuero y piel) que registran una dinámica decreciente en su participación en el crecimiento económico de la manufactura. Esto es reflejo de un sector que tiene una estructura productiva heterogénea, lo que genera un comportamiento desigual, una parte de estas actividades se encuentran integradas al sector externo, donde predominan empresas grandes y en su mayoría maquiladoras, y otra donde abundan micro y pequeñas empresas con serios problemas estructurales (García, 2004, pp. 75-76), todo ello ha influido en su lenta participación en el crecimiento del sector en los últimos años.

Más allá de presentar las estimaciones puntuales, es importante mencionar que el formato de los cuadros anexos tiene la finalidad de presentar una visión global del comportamiento de cada una de las ramas o actividades analizadas durante el periodo $\mathrm{i}$.

\section{Coeficiente de Especialización o Localización (CE)}

El coeficiente de especialización o localización permite identificar la importancia de una actividad en una zona. A diferencia del IEPB, el CE conceptualmente no considera un año base para su estimación, aunque puede ser el parámetro de comparación. Su interpretación en general resulta sencilla: cuando el coeficiente asume un valor superior a la unidad, se presenta una fuerte presencia global de esa actividad; cuando el valor es menor a la unidad, se asume que se está subespecializado (Dávila, 2002, p. 14). Más allá del resultado numérico, su interpretación permite detectar ventajas comparativas con respecto a las actividades con los GUTIERRÉZ-LAGUNES M., ROMO-OROZCO J. M., QUINTANA-ESTRADA A. 
ÍNDICE DE ESPECIALIZACIÓN PUNTO BASE Y COEFICIENTE DE ESPECIALIZACIÓN: ANÁLISIS COMPARATIVO Y ESTADÍSTICO

que se compite. El coeficiente de especialización relativa se obtiene mediante la siguiente formulación:

$$
C E_{i j}=\frac{\frac{V_{i j}}{\sum_{j=1}^{n} V_{i j}}}{\frac{\sum_{i=1}^{n} V_{i j}}{\sum_{i=1}^{n} \sum_{j=1}^{n} V_{i j}}}
$$

Donde:

$C E_{i j}=$ Coeficiente de especialización de la actividad i.

$V_{i j}=$ actividad $\mathrm{i}$ en cada periodo.

$\sum_{j=1}^{n} V_{i j}=$ total de las actividades en el periodo $\mathrm{j}$.

$\sum_{i=1}^{n} V_{i j}=$ total de la actividad i en la totalidad de los periodos.

$\sum_{i=1}^{n} V_{i j}=$ total de las actividades de los periodos analizados.

\section{Análisis de los resultados - CE}

Aplicado al sector manufacturero, el CE permite conocer el avance que tuvo cada actividad durante cada trimestre, con base en el total de los periodos analizados. Para realizar la comparación metodológica, se utilizó la misma información estadística trimestral de los 21 subsectores que componen la actividad del sector manufacturero mexicano. En el periodo de estudio del PIB del sector manufacturero, los principales resultados de estos coeficientes fueron:

- El mejor subsector fue el 334: Fabricación de equipo de computación, comunicación, medición y de otros equipos (1.600), alcanzado en el tercer trimestre del 2000. El peor subsector fue el 336: Fabricación de equipo de transporte (0.631), alcanzado en el segundo trimestre de 2009.

- El mejor desempeño en función del promedio trimestral se logró en el tercer trimestre del 2000 (1.090) y el peor desempeño fue en el primer trimestre del 2014 (0.933). En la figura 3, se puede observar la comparación de los 21 subsectores, doce de ellos tuvieron mejor coeficiente promedio que la media general (1.002). 
ÍNDICE DE ESPECIALIZACIÓN PUNTO BASE Y COEFICIENTE DE ESPECIALIZACIÓN: ANÁLISIS COMPARATIVO Y ESTADÍSTICO

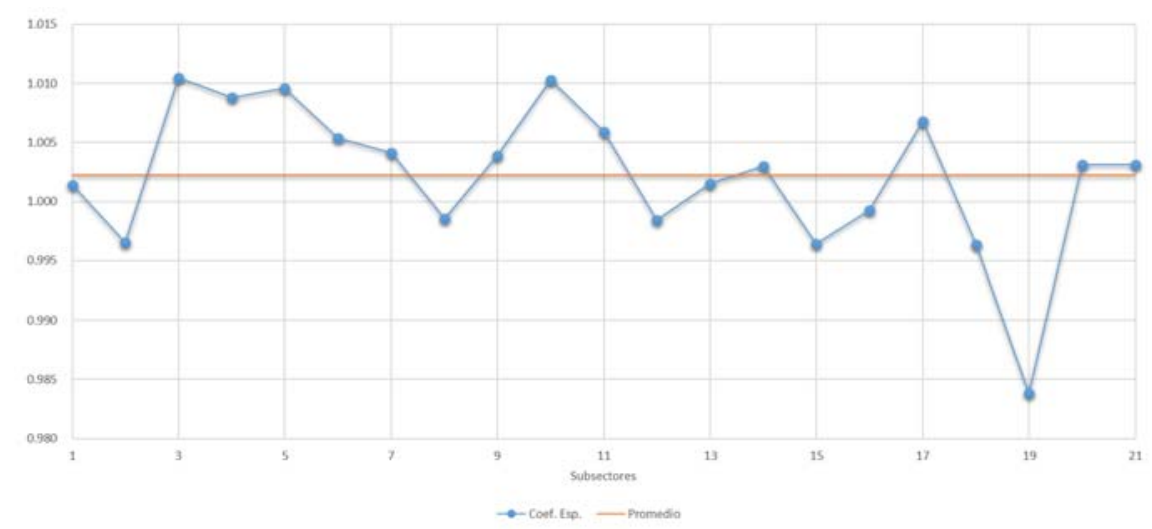

Figura 3. Desempeño de subsectores según CE.

Fuente: Elaborado con información obtenida del anexo, cuadro 2.

Los resultados presentados en la figura 4, dan cuenta de una clara reconversión de las principales ramas productivas.

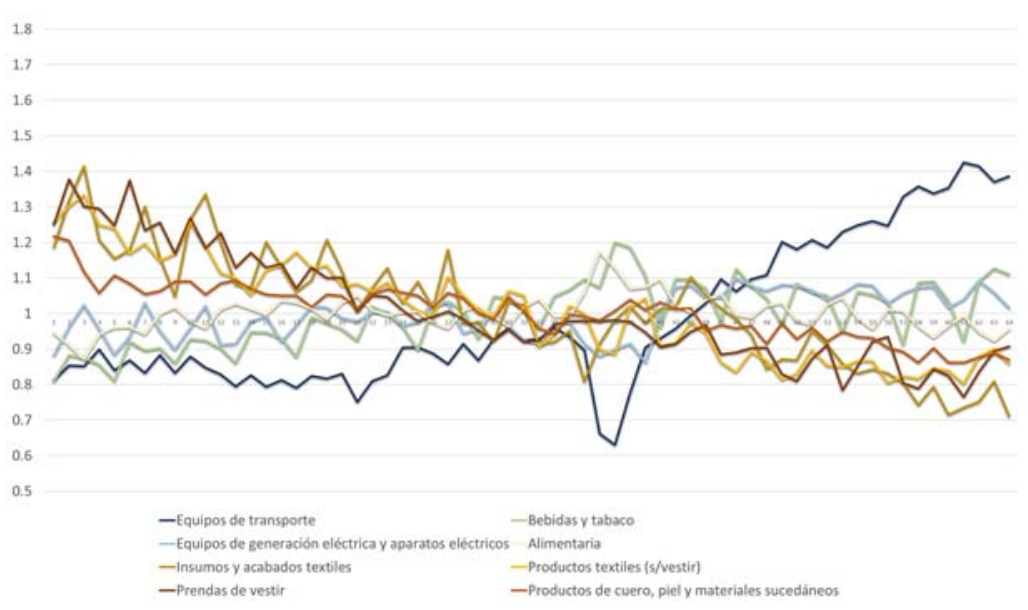

Figura 4. Coeficiente de especialización. México, selección de subsectores de la actividad manufacturera. Información trimestral, 2000-2015.

Fuente: Elaborado con información obtenida del anexo, cuadro 2.

Al inicio de la década pasada, el sector manufacturero se especializaba en la industria de la madera (321), fabricación de equipos de computación, comunicación, medición y de otros equipos, componentes y accesorios electrónicos (334) y los subsectores de la industria de insumos y productos textiles (313 y 314); debido al comportamiento observado, éste parece tener una inercia a partir del 2001, donde estos subsectores alcanzan valores pico en el GUTIERRÉZ-LAGUNES M., ROMO-OROZCO J. M., QUINTANA-ESTRADA A. 
ÍNDICE DE ESPECIALIZACIÓN PUNTO BASE Y COEFICIENTE DE ESPECIALIZACIÓN: ANÁLISIS COMPARATIVO Y ESTADÍSTICO

coeficiente. A partir de la década de los 2010 se presenta un claro declive en su participación en el PIB del sector, de manera que, en conjunto con la industria petrolera, son los que se encuentran más subespecializados para el 2015.

En contraste, el buen desempeño se tiene en los subsectores de la fabricación de equipos de transporte (336) y la industria de las bebidas y el tabaco (312), pues mostrando una baja contribución al PIB manufacturero en el 2000, al finalizar el 2015 son las actividades que, en el conjunto analizado, alcanzaron el más alto coeficiente de especialización, gracias al dinamismo y de la importancia que tienen en la actualidad, con los pros y contras que esto puede representar.

\section{RESULTADOS}

Para conocer si existe diferencia en utilizar el IEPB o el CE, se realizó una prueba de hipótesis de muestras apareadas (Kelmansky, 2009; Newbold, Carlson y Thorne, 2012; Ross, 2009). La característica distintiva de las muestras apareadas es que para cada observación del primer grupo, hay una observación relacionada en el segundo grupo. En este caso, se tiene como referencia las 21 actividades económicas que componen el sector manufacturero mexicano. El periodo de estudio se hizo del 2000 al 2015 con 64 datos trimestrales obtenidos del Instituto Nacional de Estadística, Geografía e Informática (INEGI, 2017). Para comparar los dos grupos de las 21 actividades económicas, donde el grupo A es el IEPB, y el grupo B es el CE, se aplicó la prueba de Kolmogorov-Smirnov (Zaiontz, 2017) para validar la normalidad de estos índices ${ }^{1}$; con el objeto de utilizar la t-student se establecieron las siguientes hipótesis:

Hipótesis nula: $H_{0}: \mu_{A}-\mu_{B}=0$

Hipótesis Alternativa: $H_{a}: \mu_{A}-\mu_{B} \neq 0$

Para un nivel de significancia $\alpha=5 \%$, se calculó el promedio de las diferencias de las dos muestras $(\bar{d})$, así como la desviación estándar de éstas $\left(S_{d}\right)$, donde $\mathrm{n}$ es el tamaño de la muestra de cada grupo.

\footnotetext{
${ }^{1}$ Se encontró que del total de las 21 actividades, el IEPB tiene una distribución normal en 20 de ellas, y 19 el CE.

GUTIERRÉZ-LAGUNES M., ROMO-OROZCO J. M., QUINTANA-ESTRADA A.

MAYO-AGOSTO 2018. Año 24, Número 69. Págs. 366-387 
ÍNDICE DE ESPECIALIZACIÓN PUNTO BASE Y COEFICIENTE DE ESPECIALIZACIÓN: ANÁLISIS COMPARATIVO Y ESTADÍSTICO

El test estadístico observado es:

$$
t=\frac{\bar{d}}{S_{d} / \sqrt{n}}
$$

Su valor depende de la estructura de los conjuntos de datos, con una distribución $t$ con $n-1$ grados de libertad. La región de rechazo de nivel de significancia $\alpha$ para cada tipo de hipótesis alternativa es la siguiente:

\section{Tabla 1}

Hipótesis alternativa y región de rechazo de nivel $\alpha$

\begin{tabular}{cc}
\hline Tipo de hipótesis alternativa & $\begin{array}{r}\text { Región de rechazo de } \\
\text { nivel } \alpha\end{array}$ \\
\hline$H_{a}: \mu_{A}-\mu_{B} \neq 0$ & $|T| \geq t_{20,0.05 / 2}$ \\
$H_{a}: \mu_{A}-\mu_{B}>0$ & $T \geq t_{20,0.05 / 2}$ \\
$H_{a}: \mu_{A}-\mu_{B}<0$ & $T \leq t_{20,0.05 / 2}$ \\
\hline
\end{tabular}

Fuente: Elaboración propia.

Al realizar un test $t$ para diferencia de medias con muestras apareadas usando el paquete estadístico SPSS, el resultado fue el siguiente:

\section{Tabla 2}

Estadísticas de muestras emparejadas

\begin{tabular}{llllcrr}
\hline & & Media & $\mathrm{N}$ & $\begin{array}{c}\text { Desviación } \\
\text { estándar }\end{array}$ & $\begin{array}{c}\text { Media de error } \\
\text { estándar }\end{array}$ & $\begin{array}{c}\text { Diferencia de } \\
\text { las medias }\end{array}$ \\
\hline Par 1 & CE_1 & 1.001402 & 64 & 0.0494510 & 0.0061814 & -0.05708 \\
& IEPB_1 & 1.058482 & 64 & 0.0522698 & 0.0065337 & \\
Par 2 & CE_2 & 0.996528 & 64 & 0.0904427 & 0.0113053 & -0.23421 \\
& IEPB_2 & 1.230736 & 64 & 0.1116989 & 0.0139624 & \\
Par 3 & CE_3 & 1.010451 & 64 & 0.1632758 & 0.0204095 & 0.15869 \\
& IEPB_3 & 0.851757 & 64 & 0.1376330 & 0.0172041 &
\end{tabular}


ÍNDICE DE ESPECIALIZACIÓN PUNTO BASE Y COEFICIENTE DE ESPECIALIZACIÓN: ANÁLISIS COMPARATIVO Y ESTADÍSTICO

\begin{tabular}{|c|c|c|c|c|c|c|}
\hline \multirow[t]{2}{*}{ Par 4} & CE_4 & 1.008803 & 64 & 0.1434662 & 0.0179333 & 0.20695 \\
\hline & IEPB_4 & 0.801849 & 64 & 0.1140344 & 0.0142543 & \\
\hline \multirow[t]{2}{*}{ Par 5} & CE_5 & 1.009616 & 64 & 0.1549877 & 0.0193735 & 0.20203 \\
\hline & IEPB_5 & 0.807584 & 64 & 0.1239734 & 0.0154967 & \\
\hline \multirow[t]{2}{*}{ Par 6} & CE_6 & 1.005385 & 64 & 0.0781808 & 0.0097726 & 0.17978 \\
\hline & IEPB_6 & 0.825600 & 64 & 0.0642004 & 0.0080251 & \\
\hline \multirow[t]{2}{*}{ Par 7} & CE_7 & 1.004126 & 64 & 0.1239946 & 0.0154993 & 0.33536 \\
\hline & IEPB_7 & 0.668768 & 64 & 0.0825828 & 0.0103229 & \\
\hline \multirow[t]{2}{*}{ Par 8} & CE_8 & 0.998543 & 64 & 0.0569544 & 0.0071193 & -0.12883 \\
\hline & IEPB_8 & 1.127370 & 64 & 0.0643024 & 0.0080378 & \\
\hline \multirow[t]{2}{*}{ Par 9} & CE_9 & 1.003872 & 64 & 0.1188560 & 0.0148570 & 0.08357 \\
\hline & IEPB_9 & 0.920303 & 64 & 0.1089616 & 0.0136202 & \\
\hline \multirow[t]{2}{*}{ Par 10} & CE_10 & 1.010299 & 64 & 0.1321325 & 0.0165166 & 0.08287 \\
\hline & IEPB_10 & 0.927433 & 64 & 0.1212948 & 0.0151619 & \\
\hline \multirow[t]{2}{*}{ Par 11} & CE_11 & 1.005914 & 64 & 0.0801062 & 0.0100133 & 0.05300 \\
\hline & IEPB_11 & 0.952918 & 64 & 0.0758858 & 0.0094857 & \\
\hline \multirow[t]{2}{*}{ Par 12} & CE_12 & 0.998419 & 64 & 0.0361641 & 0.0045205 & 0.06300 \\
\hline & IEPB_12 & 0.935422 & 64 & 0.0338822 & 0.0042353 & \\
\hline \multirow[t]{2}{*}{ Par 13} & CE_13 & 1.001521 & 64 & 0.0643470 & 0.0080434 & -0.14600 \\
\hline & IEPB_13 & 1.147518 & 64 & 0.0737271 & 0.0092159 & \\
\hline \multirow[t]{2}{*}{ Par 14} & CE_14 & 1.002981 & 64 & 0.0628207 & 0.0078526 & 0.11109 \\
\hline & IEPB_14 & 0.891889 & 64 & 0.0558626 & 0.0069828 & \\
\hline \multirow[t]{2}{*}{ Par 15} & CE_15 & 0.996442 & 64 & 0.0591341 & 0.0073918 & 0.00196 \\
\hline & IEPB_15 & 0.994483 & 64 & 0.0590178 & 0.0073772 & \\
\hline \multirow[t]{2}{*}{ Par 16} & CE_16 & 0.999265 & 64 & 0.0923246 & 0.0115406 & 0.00202 \\
\hline & IEPB_16 & 0.997245 & 64 & 0.0921380 & 0.0115172 & \\
\hline \multirow[t]{2}{*}{ Par 17} & CE_17 & 1.006769 & 64 & 0.1861420 & 0.0232677 & 0.28878 \\
\hline & IEPB_17 & 0.717985 & 64 & 0.1327486 & 0.0165936 & \\
\hline \multirow[t]{2}{*}{ Par 18} & CE_18 & 0.996356 & 64 & 0.0637361 & 0.0079670 & -0.13380 \\
\hline & IEPB_18 & 1.130159 & 64 & 0.0722954 & 0.0090369 & \\
\hline \multirow[t]{2}{*}{ Par 19} & CE_19 & 0.983844 & 64 & 0.2044023 & 0.0255503 & -0.22595 \\
\hline & IEPB_19 & 1.209796 & 64 & 0.2513459 & 0.0314182 & \\
\hline \multirow[t]{2}{*}{ Par 20} & CE_20 & 1.003100 & 64 & 0.0582081 & 0.0072760 & 0.04547 \\
\hline & IEPB_20 & 0.957630 & 64 & 0.0555696 & 0.0069462 & \\
\hline
\end{tabular}


ÍNDICE DE ESPECIALIZACIÓN PUNTO BASE Y COEFICIENTE DE ESPECIALIZACIÓN: ANÁLISIS COMPARATIVO Y ESTADÍSTICO

\begin{tabular}{lllllll}
\hline Par 21 & CE_21 & 1.003100 & 64 & 0.0582081 & 0.0072760 & 0.04547 \\
& IEPB_21 & 0.957630 & 64 & 0.0555696 & 0.0069462 & \\
\hline
\end{tabular}

Fuente: Elaboración propia.

De la tabla anterior, el promedio de las diferencias es $\bar{d}=0,044485$

La desviación estándar de las diferencias es $S_{d}=0.157726$

Por lo que t calculada es: $t=\frac{\bar{d}}{s_{d} / \sqrt{n}}=1.292$

Obteniendo la t-student con 20 grados de libertad, y un nivel de significancia del 5\%, en una cola, se tienen los siguientes valores:

\section{Distribución T- Student}

\begin{tabular}{|c|c|c|}
\hline$d f$ & Media & Varianza \\
\hline 20 & 0 & 1.111111 \\
\hline
\end{tabular}

\begin{tabular}{|c|c|c|c|c|c|}
\hline & $10 \%$ & $5 \%$ & $2.5 \%$ & $1 \%$ & $0.5 \%$ \\
\hline & 1.3 & 1.7 & 2.0 & 2.5280 & 2.84 \\
\hline & 1.2816 & 1.6449 & 1.9600 & 2.3263 & $2.575 \varepsilon$ \\
\hline
\end{tabular}

Por lo que, $\mathrm{t}=1.292<t_{20,0.05 / 2}=2.086$, lo cual implica que se acepta la hipótesis nula; es decir, no hay diferencia entre usar el IEPB o el CE.

Por lo tanto, el IEPB puede ser usado para establecer tendencias de actividades productivas de manera similar al CE, en todos los casos la media de las diferencias entre pares no es significativa.

Al aplicarse a los diferentes subsectores del sector manufacturero mexicano se pudieron observar comportamientos similares; los picos positivos y negativos se presentan en los mismos puntos de referencia, dando cuenta de una tendencia similar (ver figura 5). 
ÍNDICE DE ESPECIALIZACIÓN PUNTO BASE Y COEFICIENTE DE ESPECIALIZACIÓN: ANÁLISIS COMPARATIVO Y ESTADÍSTICO

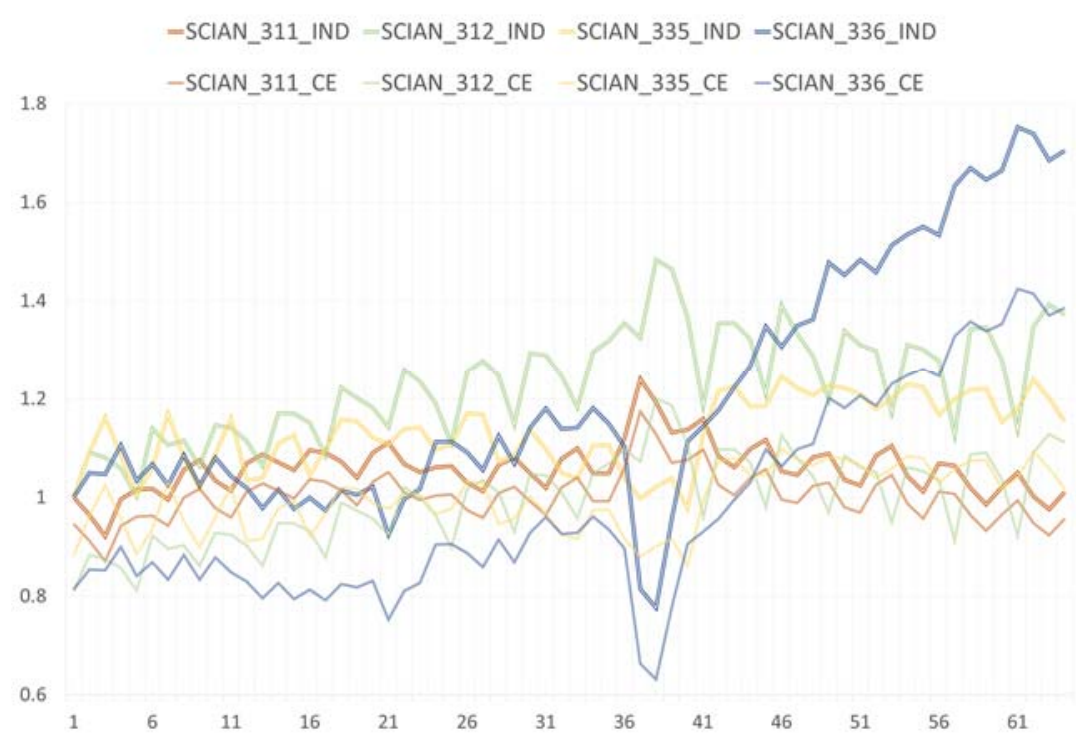

Figura 5. Comparativo del IEPB y CE. Mejor y peor desempeño entre subsectores seleccionados.

Fuente: Elaborado con información obtenida de los cuadros 1 y 2, (ANEXO).

Sin embargo, cabe señalar algunas precisiones que marcan diferencias: en la metodología del IEPB, todos los sectores analizados tienen siempre el mismo origen; es decir, la unidad y los valores siguientes dan cuenta del comportamiento particular (ver figura 5, grupo de 4 líneas que se encuentran en la parte superior); en el CE, éste depende de la participación, 0 comportamiento del sector en un momento, o territorio específico (grupo de cuatro líneas de la parte inferior). Sin embargo, en la metodología propuesta por Gutiérrez-Lagunes (2010, 2012, 2014a y 2014b), al tomar un valor de referencia que se estandariza en la unidad, no es tan fácil observar acontecimientos globales como la contracción de la crisis financiera del 2008, o la transferencia en las actividades productivas que resultan prioritarias para la economía nacional (ver figura 6, izquierda); en contraparte, con el CE, eventos como los referidos pueden evidenciarse con mayor claridad (ver figura 6, derecha). 
ÍNDICE DE ESPECIALIZACIÓN PUNTO BASE Y COEFICIENTE DE ESPECIALIZACIÓN: ANÁLISIS COMPARATIVO Y ESTADÍSTICO
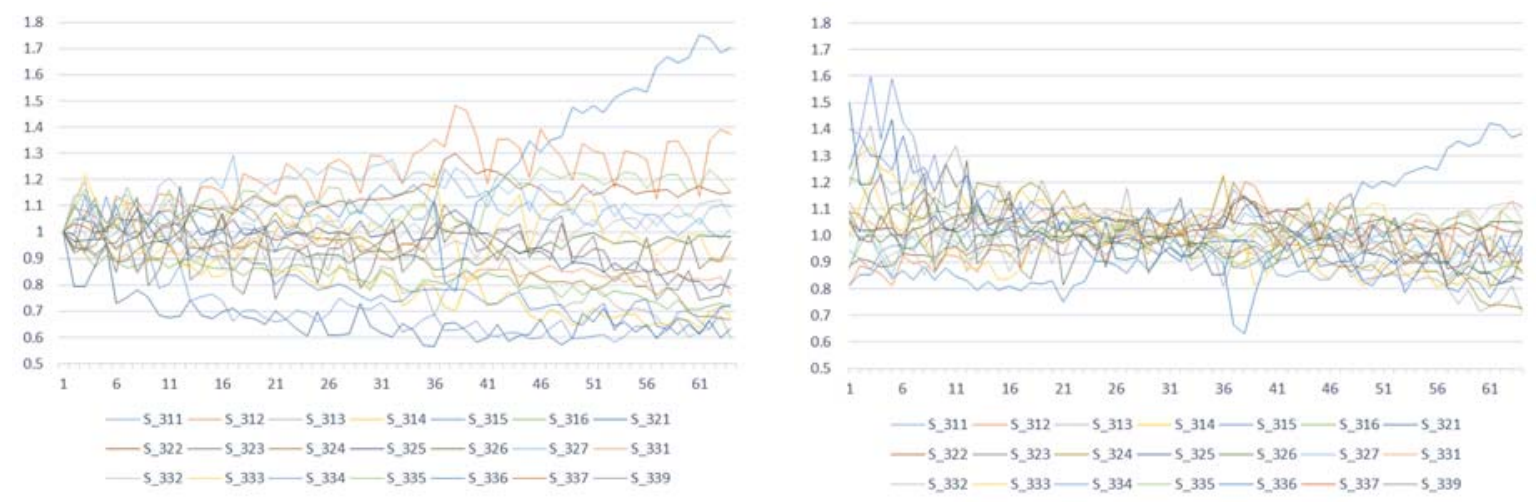

Figura 6. Comparativo del IEPB y CE. Desempeño entre subsectores.

Fuente: Elaborado con información obtenida del anexo, cuadro 1 y 2.

\section{CONCLUSIONES}

El análisis presentado demuestra que el IEPB es consistente con los conceptos de especialización referidos en la literatura, ya que estadísticamente su uso es indistinto al CE.

En el caso del IEPB que parte de un planteamiento de carácter absoluto, al relativizar en el paso siguiente, tomando en este caso el PIB total como referente, se consigue un valor que permite contrastar las metodologías y llevar a resultados similares.

El IEPB marca las tendencias de cada sector con mayor precisión por iniciar el proceso de comparación con un año base; en el análisis del crecimiento, muestra la directriz de cada rama o sector a través de rangos más amplios y valores más altos; pero no refleja la contribución inicial, lo que puede ser una debilidad si se compara directamente.

Se puede establecer una comparación, pero debe matizarse en función de que la propuesta presentada es, en origen, un índice absoluto, mientras que el coeficiente de especialización contra el que se compara es relativo. Sin embargo, a pesar de ser estadísticamente indistintos, en este caso se considera que la relación que existe entre ambos procedimientos es de complementariedad. Para análisis sectoriales, podría resultar bastante esclarecedor el IEPB, análisis que se ve reforzado y ampliado cuando se recurre también al CE. 
ÍNDICE DE ESPECIALIZACIÓN PUNTO BASE Y COEFICIENTE DE ESPECIALIZACIÓN: ANÁLISIS COMPARATIVO Y ESTADÍSTICO

\section{REFERENCIAS BIBLIOGRÁFICAS}

Cuadrado Roura, J. \& Maroto-Sánchez, A. (2012). Análisis del proceso de especialización regional en servicios en España. EURE 38(114), pp. 5-34, Recuperado de: http://www.eure.cl/index.php/eure/article/view/156.

Centro de Estudios de las Finanzas Públicas-Cámara de Diputados- (CEFP). (2005). Evolución del sector manufacturero de México 1980-2003. Recuperado de: http://www.cefp.gob.mx/intr/edocumentos/pdf/cefp/cefp0022005.pdf.

Dávila, A. (2002). Matriz de insumo-producto de la economía de Coahuila e identificación de sus flujos intersectoriales más importantes. Economía Mexicana. Nueva época, XI(1), pp. 79162.

García, M. (2004). Panorama crítico para la industria textil y del vestido mexicana. El Cotidiano, 20(127), pp. 73-84.

Pérez, M., Gordo E. \& Gil M. (2003). Los efectos de la integración económica sobre la especialización y distribución geográfica de la actividad industrial en los países de la UE. Banco de España, (3), pp. 1-62.

Gutiérrez, M. (2010). La sectorización económica y su vinculación con la probabilidad de incumplimiento. Administración, Finanzas y Economía. 4(2), pp. 93-110.

Gutiérrez, M. (2012). Desarrollo de las ramas económicas en México. Periodo 2004 - primer semestre 2011. Eseconomía. (33), pp. 79-104.

Gutiérrez, M. (2014). La evolución de la industria mexicana a partir de las ramas económicas y su relación con el comercio exterior. Revista Paradigma Económico, 6(1), pp. 51-74. Recuperado

de: http://web.uaemex.mx/feconomia/Publicaciones/p601/03_PARADIG_FINAL_4.pdf.

Gutiérrez, M. (2014). Un estudio exploratorio sobre el crecimiento de industria mexicana y el comercio exterior. Periodo 2004-2012. Eseconomía, IX(41), pp. 75-104. Recuperado de: http://www.proyectos.ese.ipn.mx/. 
ÍNDICE DE ESPECIALIZACIÓN PUNTO BASE Y COEFICIENTE DE ESPECIALIZACIÓN: ANÁLISIS COMPARATIVO Y ESTADÍSTICO

Instituto Nacional de Estadística y Geografía - INEGI (2017). Banco de Información Económica. Disponible en la base de datos del INEGI.

INEGI (2013). Sistema de Clasificación Industrial de América del Norte 2013. Disponible en la base de datos del INEGI.

Kelmansky, D. (2009). Estadística para todos. Argentina: Artes Gráficas Rioplatense.

Newbold, P., Carlson, W. \& Thorne, B. (2012). Statistics for Business and Economics. EUA: Pearson Prentice Hall.

Quiroga, R. (2001). Indicadores de sostenibilidad ambiental y de desarrollo sostenible: estado del arte y perspectivas. Serie manuales. Recuperado de: http://repositorio.cepal.org/bitstream/handle/11362/5570/S0110817_es.pdf?sequence=1.

Ross, S. (2009). Introductory statistics. EUA: Elsevier Science.

Sepúlveda, S. (2008). Metodología para estimar el nivel de desarrollo sostenible de territorios. Costa Rica: Instituto Interamericano de Cooperación para la Agricultura.

Yizhou, Z. \& Méndez, E. (2007). Técnicas de análisis regional aplicadas en tres regiones del Oriente de China. Recuperado de: http://www.eumed.net/libros-gratis/2007b/287/.

Vázquez, R. (2015). Concentración empresarial y cambio estructural: alimentos, bebidas y tabaco en México. Revista Problemas del Desarrollo, 46(180), pp. 52-76.

Zaiontz, C. (2017). Kolmogorov-Smirnov Test for Normality. Real Statistics Using Excel. Recuperado de http://www.real-statistics.com/tests-normality-and-symmetry/statisticaltests-normality-symmetry/kolmogorov-smirnov-test/. 
ÍNDICE DE ESPECIALIZACIÓN PUNTO BASE Y COEFICIENTE DE ESPECIALIZACIÓN: ANÁLISIS COMPARATIVO Y ESTADÍSTICO

\section{ANEXO}

Subsectores que integran el sector manufacturero de acuerdo al Sistema de Clasificación Industrial de América del Norte 2013 (SCIAN) (INEGI, 2013):

\begin{tabular}{lll}
\hline & SCIAN* & \\
\hline 1 & 311 & Industria alimentaria. \\
2 & 312 & Industria de las bebidas y del tabaco. \\
3 & 313 & Fabricación de insumos textiles y acabado de textiles. \\
4 & 314 & Fabricación de productos textiles, excepto prendas de vestir. \\
5 & 315 & Fabricación de prendas de vestir. \\
6 & 316 & Curtido y acabado de cuero y piel, y fabricación de productos de cuero, piel y \\
7 & 321 & Industria de la madera. \\
8 & 322 & Industria del papel. \\
9 & 323 & Impresión e industrias conexas. \\
10 & 324 & Fabricación de productos derivados del petróleo y carbón. \\
11 & 325 & Industria química. \\
12 & 326 & Industria del plástico y del hule. \\
13 & 327 & Fabricación de productos a base de minerales no metálicos. \\
14 & 331 & Industrias metálicas básicas. \\
15 & 332 & Fabricación de productos metálicos. \\
16 & 333 & Fabricación de maquinaria y equipo. \\
17 & 334 & Fabricación de equipo de computación, comunicación, medición y de otros \\
18 & 335 & Fabricación de equipo de generación eléctrica y aparatos eléctricos. \\
21 & 336 & Fabricación de equipo de transporte. \\
21 & 337 & Fabricación de muebles, colchones y persianas. \\
\hline & 339 & Otras industrias manufactureras. \\
\hline
\end{tabular}

La numeración de cada subsector resulta útil para identificar el nombre de cada uno de ellos en la primera fila, en los cuadros 1 y 2 a continuación presentados: 
ÍNDICE DE ESPECIALIZACIÓN PUNTO BASE Y COEFICIENTE DE ESPECIALIZACIÓN: ANÁLISIS COMPARATIVO Y ESTADÍSTICO

Cuadro 1. Índice de especialización punto base (IEPB). México, 21 subsectores de la actividad manufacturera. Información trimestral, 2000-2015.

\begin{tabular}{|c|c|c|c|c|c|c|c|c|c|c|c|c|c|c|c|c|c|c|c|c|c|}
\hline FECHA & 1 & 2 & 3 & 4 & $\begin{array}{l}1 \\
\end{array}$ & 6 & 7 & 8 & 9 & 10 & 11 & 12 & 13 & 14 & 15 & 16 & 17 & 18 & 19 & 20 & 21 \\
\hline 2000/03 & 1.00 & 1.00 & 1.00 & 1.00 & 1.00 & 1.00 & 1.00 & 1.00 & 1.00 & 1.00 & 1.00 & 1.00 & 1.00 & 1.00 & 1.00 & 1.00 & 1.00 & 1.00 & 1.00 & 1.00 & 1.00 \\
\hline $2000 / 06$ & 0.96 & 1.09 & 1.11 & 1.04 & 1.10 & 0.99 & 0.80 & 1.03 & 0.93 & 0.99 & 0.96 & 0.92 & 1.14 & 0.95 & 1.02 & 1.11 & 0.99 & 1.09 & 1.05 & 0.94 & 0.94 \\
\hline $2000 / 09$ & 0.92 & 1.08 & 1.19 & 1.06 & 1.04 & 0.92 & 0.79 & 1.02 & 1.07 & 0.93 & 0.97 & 0.94 & 1.14 & 0.93 & 1.00 & 1.22 & 1.14 & 1.16 & 1.05 & 0.93 & 0.93 \\
\hline $2000 / 12$ & 1.00 & 1.06 & 1.02 & 0.99 & 1.04 & 0.87 & 0.87 & 1.00 & 1.13 & 0.95 & 0.97 & 0.91 & 1.06 & 0.91 & 0.90 & 1.12 & 0.97 & 1.09 & 1.11 & 1.03 & 1.03 \\
\hline $2001 / 03$ & 1.02 & 1.00 & 0.97 & 0.98 & 1.00 & 0.91 & 0.96 & 1.00 & 0.96 & 1.02 & 0.98 & 0.94 & 0.97 & 0.94 & 0.93 & 1.03 & 1.13 & 1.00 & 1.03 & 0.96 & 0.96 \\
\hline $2001 / 06$ & 1.02 & 1.14 & 0.99 & 0.93 & 1.10 & 0.89 & 0.73 & 1.05 & 0.85 & 1.03 & 0.94 & 0.89 & 1.12 & 0.89 & 0.95 & 1.01 & 1.02 & 1.06 & 1.07 & 0.96 & 0.96 \\
\hline $2001 / 09$ & 1.00 & 1.11 & 1.10 & 0.95 & 0.99 & 0.87 & 0.75 & 1.05 & 1.06 & 0.99 & 0.97 & 0.90 & 1.14 & 0.92 & 0.95 & 1.13 & 0.98 & 1.17 & 1.02 & 1.02 & 1.02 \\
\hline $2001 / 12$ & 1.06 & 1.12 & 0.98 & 0.91 & 1.00 & 0.87 & 0.78 & 1.05 & 1.13 & 0.98 & 0.98 & 0.93 & 1.09 & 0.85 & 0.85 & 0.98 & 0.85 & 1.08 & 1.09 & 1.09 & 1.09 \\
\hline $2002 / 03$ & 1.08 & 1.06 & 0.88 & 0.93 & 0.93 & 0.90 & 0.75 & 1.03 & 0.80 & 1.05 & 1.02 & 0.96 & 1.02 & 0.91 & 0.93 & 0.89 & 0.93 & 1.02 & 1.02 & 0.98 & 0.98 \\
\hline $2002 / 06$ & 1.03 & 1.15 & 1.07 & 1.00 & 1.01 & 0.90 & 0.68 & 1.07 & 0.86 & 1.02 & 0.97 & 0.97 & 1.18 & 0.90 & 0.98 & 0.93 & 0.79 & 1.09 & 1.08 & 1.01 & 1.01 \\
\hline $2002 / 09$ & 1.01 & 1.14 & 1.13 & 0.95 & 0.95 & 0.87 & 0.68 & 1.08 & 0.97 & 1.04 & 0.97 & 0.95 & 1.20 & 0.93 & 0.98 & 0.96 & 0.80 & 1.16 & 1.04 & 1.03 & 1.03 \\
\hline $2002 / 12$ & 1.07 & 1.11 & 1.01 & 0.89 & 0.98 & 0.89 & 0.68 & 1.04 & 1.17 & 0.96 & 1.00 & 0.88 & 1.16 & 0.91 & 0.86 & 0.87 & 0.88 & 1.03 & 1.02 & 1.03 & 1.03 \\
\hline $2003 / 03$ & 1.09 & 1.06 & 0.91 & 0.87 & 0.90 & 0.90 & 0.74 & 1.07 & 0.82 & 1.10 & 1.07 & 0.94 & 1.07 & 0.96 & 0.97 & 0.87 & 0.74 & 1.04 & 0.98 & 0.99 & 0.99 \\
\hline $2003 / 06$ & 1.07 & 1.17 & 0.91 & 0.84 & 0.94 & 0.88 & 0.68 & 1.09 & 0.86 & 1.09 & 0.99 & 0.94 & 1.19 & 0.93 & 0.97 & 0.90 & 0.76 & 1.11 & 1.02 & 0.94 & 0.94 \\
\hline $2003 / 09$ & 1.05 & 1.17 & 1.01 & 0.89 & 0.90 & 0.87 & 0.67 & 1.09 & 0.97 & 1.09 & 1.00 & 0.95 & 1.21 & 0.94 & 0.97 & 0.83 & 0.76 & 1.13 & 0.98 & 0.98 & 0.98 \\
\hline $2003 / 12$ & 1.10 & 1.15 & 0.95 & 0.90 & 0.91 & 0.86 & 0.70 & 1.09 & 1.07 & 1.00 & 1.01 & 0.96 & 1.16 & 0.92 & 0.86 & 0.84 & 0.74 & 1.04 & 1.00 & 1.07 & 1.07 \\
\hline $2004 / 03$ & 1.09 & 1.08 & 0.89 & 0.93 & 0.86 & 0.86 & 0.71 & 1.07 & 0.80 & 1.08 & 1.05 & 0.94 & 1.29 & 0.94 & 0.95 & 0.87 & 0.66 & 1.10 & 0.97 & 0.95 & 0.95 \\
\hline $2004 / 06$ & 1.07 & 1.22 & 0.92 & 0.90 & 0.90 & 0.84 & 0.68 & 1.11 & 0.77 & 1.10 & 0.99 & 0.93 & 1.07 & 0.95 & 1.03 & 0.96 & 0.70 & 1.16 & 1.01 & 0.97 & 0.97 \\
\hline $2004 / 09$ & 1.04 & 1.20 & 1.02 & 0.90 & 0.88 & 0.87 & 0.67 & 1.14 & 0.89 & 1.08 & 1.00 & 0.97 & 1.13 & 0.98 & 1.03 & 0.93 & 0.70 & 1.15 & 1.01 & 1.01 & 1.01 \\
\hline $2004 / 12$ & 1.09 & 1.18 & 0.94 & 0.86 & 0.88 & 0.86 & 0.65 & 1.12 & 1.01 & 1.01 & 1.01 & 0.93 & 1.18 & 0.93 & 0.89 & 1.00 & 0.67 & 1.12 & 1.02 & 0.99 & 0.99 \\
\hline $2005 / 03$ & 1.11 & 1.14 & 0.85 & 0.86 & 0.81 & 0.83 & 0.70 & 1.10 & 0.75 & 1.07 & 1.05 & 0.93 & 1.19 & 0.99 & 1.01 & 1.00 & 0.66 & 1.11 & 0.92 & 0.97 & 0.97 \\
\hline $2005 / 06$ & 1.07 & 1.26 & 0.90 & 0.84 & 0.84 & 0.87 & 0.67 & 1.13 & 0.83 & 1.01 & 0.99 & 0.94 & 1.21 & 0.98 & 1.03 & 1.09 & 0.67 & 1.14 & 1.00 & 0.98 & 0.98 \\
\hline $2005 / 09$ & 1.05 & 1.23 & 0.95 & 0.86 & 0.84 & 0.88 & 0.63 & 1.13 & 0.93 & 1.03 & 1.00 & 0.93 & 1.20 & 0.95 & 1.03 & 1.01 & 0.70 & 1.14 & 1.02 & 1.00 & 1.00 \\
\hline $2005 / 12$ & 1.06 & 1.19 & 0.87 & 0.83 & 0.81 & 0.87 & 0.60 & 1.07 & 0.95 & 0.99 & 0.99 & 0.90 & 1.24 & 0.95 & 0.94 & 1.06 & 0.69 & 1.10 & 1.11 & 0.98 & 0.98 \\
\hline $2006 / 03$ & 1.06 & 1.11 & 0.92 & 0.80 & 0.78 & 0.86 & 0.70 & 1.09 & 0.81 & 0.98 & 1.02 & 0.92 & 1.22 & 0.98 & 1.04 & 0.96 & 0.66 & 1.11 & 1.11 & 0.95 & 0.95 \\
\hline $2006 / 06$ & 1.03 & 1.26 & 0.86 & 0.79 & 0.79 & 0.83 & 0.61 & 1.10 & 0.94 & 0.97 & 1.00 & 0.91 & 1.25 & 0.97 & 1.04 & 1.06 & 0.69 & 1.17 & 1.09 & 0.95 & 0.95 \\
\hline $2006 / 09$ & 1.01 & 1.28 & 0.99 & 0.88 & 0.80 & 0.87 & 0.61 & 1.12 & 0.94 & 0.97 & 1.00 & 0.91 & 1.23 & 0.95 & 1.05 & 0.99 & 0.75 & 1.17 & 1.06 & 1.00 & 1.00 \\
\hline $2006 / 12$ & 1.07 & 1.25 & 0.82 & 0.84 & 0.79 & 0.86 & 0.61 & 1.11 & 0.97 & 0.97 & 0.99 & 0.88 & 1.22 & 0.90 & 0.97 & 1.02 & 0.72 & 1.07 & 1.12 & 0.99 & 0.99 \\
\hline $2007 / 03$ & 1.08 & 1.15 & 0.82 & 0.80 & 0.76 & 0.82 & 0.73 & 1.12 & 0.79 & 0.98 & 1.04 & .91 & 1.19 & 0.95 & .02 & 0.93 & 0.71 & 1.09 & 1.07 & 0.92 & 0.92 \\
\hline $2007 / 06$ & 1.05 & 1.29 & 0.78 & 0.79 & 0.74 & 0.81 & 0.64 & 1.12 & 0.84 & 0.96 & 0.98 & 92 & 1.25 & 0.92 & .00 & 1.04 & 0.71 & 1.14 & 1.14 & 0.93 & 0.93 \\
\hline $2007 / 09$ & 1.02 & 1.29 & 0.87 & 0.85 & 0.76 & 0.86 & 0.62 & 1.12 & 0.96 & 0.93 & 0.99 & 93 & 1.26 & 0.90 & .02 & 1.01 & 0.73 & 1.10 & 1.18 & 0.97 & 0.97 \\
\hline 2007/12 & 1.08 & 1.25 & 0.86 & 0.83 & 0.74 & 83 & 0.60 & 1.13 & 1.05 & 0.92 & 1.00 & 90 & 1.28 & 0.89 & .96 & 1.01 & 0.68 & 1.05 & 1.14 & 1.01 & 1.01 \\
\hline 2008/03 & 1.10 & 1.18 & 0.77 & 0.72 & 0.74 & 0.79 & 0.65 & 1.15 & 0.85 & 0.95 & 1.04 & 91 & 1.19 & 0.95 & .99 & 1.04 & 0.62 & 1.04 & 1.14 & 0.91 & 0.91 \\
\hline $2008 / 06$ & 1.05 & 1.30 & 0.78 & 0.75 & 0.78 & 0.77 & 0.63 & 1.15 & 0.89 & 0.95 & 0.97 & .91 & 1.23 & 0.96 & .03 & 1.09 & 0.64 & 1.10 & 1.18 & 0.93 & 0.93 \\
\hline $2008 / 09$ & 1.05 & 1.32 & 0.80 & 0.81 & 0.78 & 0.82 & 0.57 & 1.18 & 1.00 & 0.92 & 0.97 & 0.90 & 1.23 & 0.93 & .00 & 1.10 & 0.67 & 1.11 & 1.15 & 0.98 & 0.98 \\
\hline $2008 / 12$ & 1.12 & 1.35 & 0.68 & 0.79 & 0.78 & 0.81 & 0.57 & 1.17 & 1.12 & 0.99 & 0.97 & 0.86 & 1.19 & 0.83 & 0.91 & 1.23 & 0.69 & 1.04 & 1.10 & 1.03 & 1.03 \\
\hline $2009 / 03$ & 1.24 & 1.32 & 0.77 & 0.71 & 0.78 & 0.81 & 0.65 & 1.28 & 0.96 & 1.10 & 1.06 & 0.86 & 1.17 & 0.83 & 0.94 & 1.01 & 0.63 & 1.00 & 0.81 & 1.01 & 1.01 \\
\hline 2009/06 & 1.19 & 1.48 & 0.83 & 0.70 & 0.78 & 0.83 & 0.66 & 1.30 & 1.02 & 1.05 & 1.08 & 89 & 1.25 & 0.84 & 0.97 & 0.97 & 0.63 & 1.02 & 0.78 & 0.99 & 0.99 \\
\hline $2009 / 99$ & $\begin{array}{l}1.13 \\
1.14\end{array}$ & $\begin{array}{l}1.46 \\
1.37\end{array}$ & $\begin{array}{l}0.86 \\
0.82\end{array}$ & $\begin{array}{l}0.81 \\
0.83\end{array}$ & $\begin{array}{l}0.78 \\
0.76\end{array}$ & $\begin{array}{l}0.85 \\
0.83\end{array}$ & $\begin{array}{l}0.63 \\
0.58\end{array}$ & $\begin{array}{l}1.26 \\
1.22\end{array}$ & $\begin{array}{l}1.04 \\
0.93\end{array}$ & $\begin{array}{l}1.03 \\
1.01\end{array}$ & $\begin{array}{l}1.05 \\
0.99\end{array}$ & $\begin{array}{l}0.90 \\
0.87\end{array}$ & $\begin{array}{l}1.22 \\
1.16\end{array}$ & $\begin{array}{l}0.82 \\
0.85\end{array}$ & 0.95 & $\begin{array}{l}0.81 \\
0.84\end{array}$ & $\begin{array}{l}0.64 \\
066\end{array}$ & 1.04 & 0.96 & 1.00 & 1.00 \\
\hline 2010/03 & 1.16 & 1.18 & 0.85 & 0.72 & 0.73 & 0.85 & 0.60 & 1.24 & 0.94 & 0.97 & 0.99 & 0.90 & 1.09 & 0.86 & 0.98 & 0.91 & 0.61 & 1.13 & 1.14 & 0.94 & 0.94 \\
\hline $2010 / 06$ & 1.08 & 1.35 & 0.86 & 0.73 & 0.73 & 0.83 & 0.65 & 1.23 & 1.01 & 0.93 & 0.97 & 0.92 & 1.18 & 0.86 & 0.99 & 1.03 & 0.60 & 1.22 & 1.18 & 0.90 & 0.90 \\
\hline $2010 / 09$ & 1.06 & 1.35 & 0.93 & 0.78 & 0.76 & 0.83 & 0.59 & 1.22 & 1.01 & 0.89 & 0.95 & 0.95 & 1.15 & 0.86 & 1.01 & 1.09 & 0.62 & 1.23 & 1.22 & 0.97 & 0.97 \\
\hline 2010/12 & 1.10 & 1.32 & 0.88 & 0.75 & 0.77 & 0.78 & 0.61 & 1.16 & 1.03 & 0.8 & 0.92 & 0.92 & 1.19 & 0.8 & 0.97 & 1.14 & 0.6 & 1.18 & 1.2 & 1.00 & 1.00 \\
\hline $2011 / 03$ & 1.12 & 1.21 & 0.86 & 0.69 & 0.71 & 0.80 & 0.61 & 1.18 & 0.90 & 0.85 & 0.94 & 0.93 & 1.08 & 0.8 & 1.01 & 0.94 & 0.5 & 1.19 & 1.35 & 0.92 & 0.92 \\
\hline $2011 / 06$ & 1.05 & 1.39 & 0.83 & 0.66 & 0.71 & 0.79 & 0.67 & 1.16 & 0.97 & 0.82 & 0.94 & 0.94 & 1.18 & 0.8 & 0.98 & 1.07 & 0.6 & 1.25 & 1.31 & 0.93 & 0.93 \\
\hline $2011 / 09$ & 1.05 & 1.33 & 0.79 & 0.71 & 0.72 & 0.79 & 0.60 & 1.13 & 1.04 & 0.81 & 0.92 & 0.96 & 1.15 & 0.8 & 1.01 & 1.11 & 0.6 & 1.22 & 1.35 & 0.90 & 0.90 \\
\hline $2011 / 12$ & 1.08 & 1.29 & 0.71 & 0.69 & 0.72 & 0.75 & 0.57 & 1.10 & 1.06 & 0.81 & 0.86 & 0.93 & 1.15 & 0.8 & 0.97 & 1.10 & 0.6 & 1.21 & 1.36 & 1.03 & 1.03 \\
\hline $2012 / 03$ & 1.09 & 1.20 & 0.73 & 0.65 & 0.66 & 0.80 & 0.60 & 1.13 & 0.88 & 0.81 & 0.89 & $0 . s^{\prime}$ & 1.06 & 0.8 & 0.99 & 1.09 & 0.6 & 1.23 & 1.48 & 0.90 & 0.90 \\
\hline $2012 / 06$ & 1.04 & 1.34 & 0.73 & 0.66 & 0.65 & 0.76 & 0.69 & 1.18 & 0.94 & 0.82 & 0.88 & 0.99 & 1.13 & 0.8 & 1.02 & 1.12 & 0.60 & 1.22 & 1.45 & 0.90 & 0.90 \\
\hline 2012/09 & 1.02 & 1.31 & 0.80 & 0.71 & 0.70 & 0.79 & 0.66 & 1.14 & 0.98 & 0.78 & 0.87 & 1.00 & 1.16 & 0.8 & 1.05 & 1.11 & 0.60 & 1.21 & 1.48 & 0.94 & 0.94 \\
\hline $2012 / 12$ & 1.08 & 1.30 & 0.77 & 0.68 & 0.73 & 0.76 & 0.71 & 1.15 & 0.85 & 0.8 & 0.85 & 0.98 & 1.13 & 0.8 & 1.01 & 0.98 & 0.61 & 1.18 & 1.46 & 0.93 & 0.93 \\
\hline 2013/03 & 1.10 & 1.17 & 0.72 & 0.68 & 0.63 & 0.78 & 0.64 & 1.18 & 0.81 & 0.8 & 0.88 & 0.95 & 1.06 & 0.8 & 1.6 & 0.95 & 0.58 & 1.20 & 1.51 & 0.86 & 0.86 \\
\hline 2013/06 & 1.04 & 1.31 & 0.70 & 0.69 & 0.68 & 0.77 & 0.66 & 1.17 & 0.85 & 0.8 & 0.86 & 0.96 & 1.11 & 0.8 & 1.05 & 1.00 & 0.61 & 1.23 & 1.54 & 0.86 & 0.86 \\
\hline $2013 / 09$ & 1.01 & 1.30 & 0.71 & 0.69 & 0.74 & 0.76 & 0.62 & 1.14 & 0.85 & 0.8 & 0.89 & 0.96 & 1.08 & 0.8 & 1.8 & 0.98 & 0.6 & 1.22 & 1.55 & 0.85 & 0.85 \\
\hline 2013/12 & 1.07 & 1.28 & 0.70 & 0.64 & 0.75 & 0.74 & 0.65 & 1.15 & 0.86 & 0.8 & 0.8 & 0. & 1.06 & 0. & 1.6 & 0.95 & 0 & 1.17 & 1.53 & 0.98 & 0.98 \\
\hline $2014 / 03$ & 1.06 & 1.13 & 0.68 & 0.65 & 0.64 & 0.73 & 0.60 & 1.16 & 0.74 & 0.7 & 0.86 & 0.96 & 1.02 & 0.90 & 1.6 & & 0 & 1.20 & 1.63 & 0.84 & 0.84 \\
\hline $2014 / 06$ & 1.02 & 1.34 & 0.63 & 0.65 & 0.63 & 0.71 & 0.65 & 1.16 & 0.79 & 0.79 & 0.80 & 18 & 1.08 & 0.8 & 1.07 & 0.94 & 0.63 & 1.22 & 1.67 & 0.84 & 0.84 \\
\hline 2014/09 & 0.99 & 1.35 & 0.67 & 0.67 & 0.68 & 0.74 & 0.61 & 1.13 & 0.78 & 0.76 & 0.84 & 7 & 1.06 & 0. & 1. & & 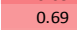 & 1.22 & 1.65 & 0.86 & 0.86 \\
\hline $2014 / 12$ & 1.02 & 1.28 & 0.60 & 0.67 & 0.66 & 0.71 & 0.65 & 1.16 & 0.85 & 0.6 & 0.82 & 0.95 & 1.0 & 0.82 & 1.6 & 0.99 & 0.70 & 1.15 & 1.66 & 0.99 & 0.99 \\
\hline $2015 / 03$ & 1.05 & 1.14 & 0.62 & 0.64 & 0.61 & 0.71 & 0.61 & 1.18 & 0.74 & 0.68 & 0.82 & 0.99 & 1.03 & 0.82 & 1. & 0.99 & 0.66 & 1.18 & 75 & 0.86 & .86 \\
\hline $2015 / 06$ & 1.00 & 1.35 & 0.63 & 0.70 & 0.67 & 0.72 & 0.66 & 1.16 & 0.75 & 0.68 & 0.78 & 0.99 & 1.09 & 0.82 & 1.12 & 0.91 & 0.63 & 1.24 & 1.74 & 0.90 & .90 \\
\hline 2015/09 & 0.98 & 1.39 & 0.68 & 0.72 & 0.72 & 0.73 & 0.60 & 1.14 & 0.76 & 0.67 & 0.80 & 0.99 & 1.10 & 0.83 & 1.12 & 0.90 & 0.72 & 1.20 & 1.68 & 0.89 & 0.89 \\
\hline $2015 / 12$ & 1.01 & 1.37 & 0.60 & 0.68 & 0.73 & 0.71 & 0.63 & 1.15 & 0.86 & 0.67 & 0.79 & 0.98 & 1.10 & 0.76 & 1.05 & 0.91 & 0.72 & 1.15 & 1.70 & 0.96 & 0.96 \\
\hline
\end{tabular}

GUTIERRÉZ-LAGUNES M., ROMO-OROZCO J. M., QUINTANA-ESTRADA A.

MAYO-AGOSTO 2018. Año 24, Número 69. Págs. 366-387 
ÍNDICE DE ESPECIALIZACIÓN PUNTO BASE Y COEFICIENTE DE ESPECIALIZACIÓN: ANÁLISIS COMPARATIVO Y ESTADÍSTICO

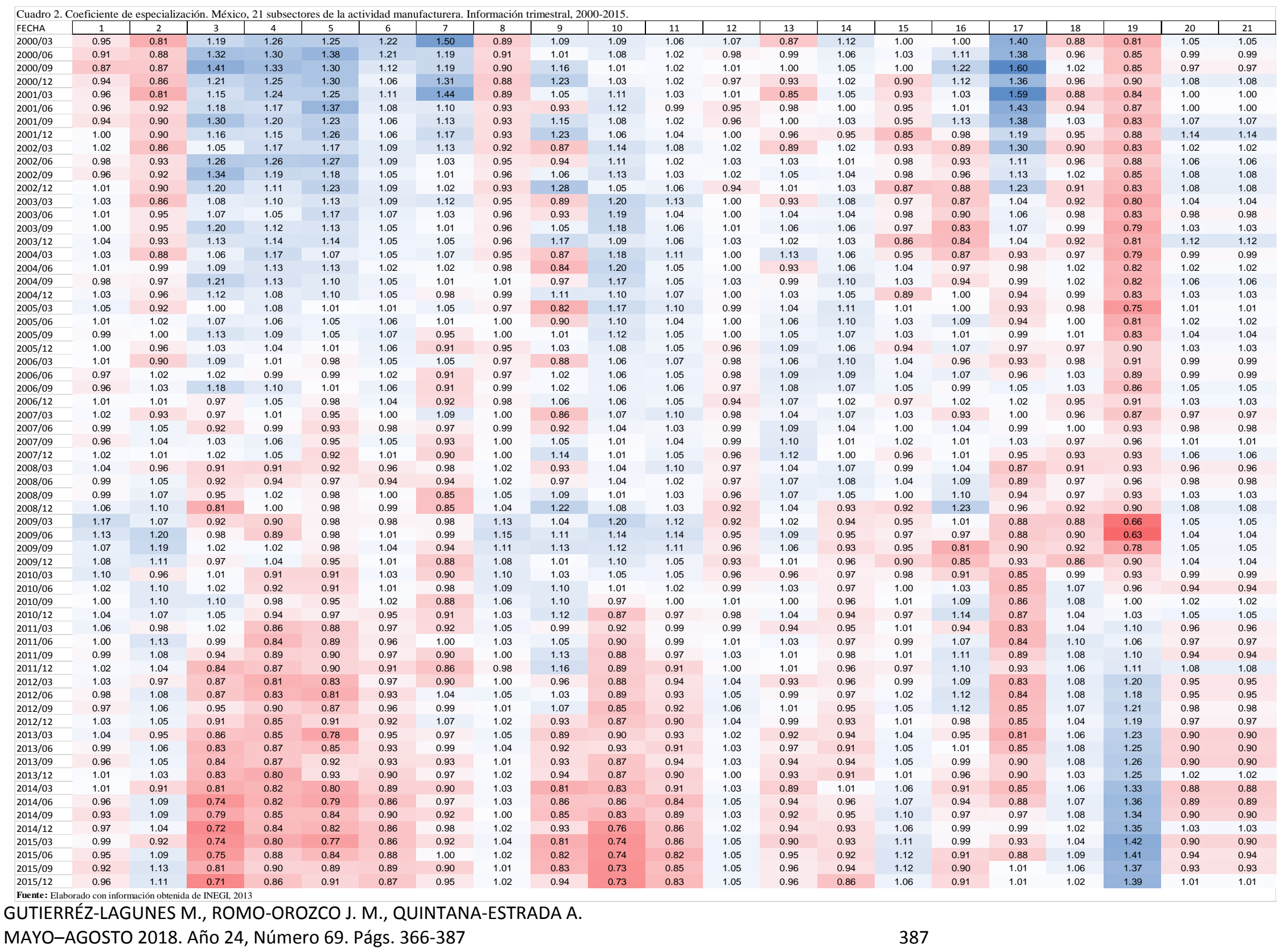

